\title{
BLACK HOLES AND SOLITONS OF THE QUANTIZED DISPERSIONLESS NLS AND DNLS EQUATIONS
}

\author{
OKTAY K. PASHAEV ${ }^{123}$ and JYH-HAO LEE ${ }^{3}$
}

(Received 28 April, 2000; revised 18 September, 2001)

\begin{abstract}
The classical dynamics of non-relativistic particles are described by the Schrödinger wave equation, perturbed by quantum potential nonlinearity. Quantization of this dispersionless equation, implemented by deformation of the potential strength, recovers the standard Schrödinger equation. In addition, the classically forbidden region corresponds to the Planck constant analytically continued to pure imaginary. values. We apply the same procedure to the NLS and DNLS equations, constructing first the corresponding dispersionless limits and then adding quantum deformations. All these deformations admit the Lax representation as well as the Hirota bilinear form. In the classically forbidden region we find soliton resonances and black hole phenomena. For deformed DNLS the chiral solitons with single event horizon and resonance dynamics are constructed.
\end{abstract}

\section{Quantization of the classical dynamics}

1.1. Classical dynamics in the Schrödinger representation The classical dynamics of non-relativistic particles can be naturally described in terms of Schrödinger-type wave equations. Thus the Hamilton-Jacobi equation

$$
-S_{t}+\left(S_{x}\right)^{2}+U=0
$$

for a non-relativistic particle $(m=1 / 2)$ in potential $U(x, t)$ combined with the Liouville equation

$$
-\rho_{t}+2\left(\rho S_{x}\right)_{x}=0
$$

\footnotetext{
'Department of Mathematics, Izmir Institute of Technology, Liyla-Izmir, 35437 Turkey; e-mail: pashaev@likya.iyte.edu.tr.

${ }^{2}$ Joint Institute for Nuclear Research, Dubna, 141980, Russian Federation.

${ }^{3}$ Institute of Mathematics, Academia Sinica, Taipei 11529, Taiwan, ROC;

e-mail: leejh@math.sinica.edu.tw.

(C) Australian Mathematical Society 2002, Serial-fee code 1446-1811/02
} 
for the density of integral invariant in the gradient dynamical system $x_{t}=-2 S_{x}$ (the defining relation for a particle's momentum), is equivalent to the modification

$$
i \psi_{t}+\psi_{x x}-U \psi=\frac{|\psi|_{x x}}{|\psi|} \psi
$$

of the Schrödinger equation by the so called "quantum potential". Here the complex wave function is given by $\psi=\sqrt{\rho} e^{-i S}$. Since (1) and (2) describe the semiclassical limit of the Schrödinger equation we call (3) its dispersionless limit. In this connection, the additional term on the right-hand side completely compensates for the $U(1)$ gauge invariant contribution from dispersion on the left-hand side.

1.2. Quantum deformation The original Schrödinger equation

$$
i \hbar \chi_{1}+\hbar^{2} \chi_{x x}-U \chi=0
$$

has to appear from quantum deformation

$$
i \psi_{t}+\psi_{x x}-U \psi=\left(1-\hbar^{2}\right) \frac{|\psi|_{x x}}{|\psi|} \psi
$$

of the dispersionless equation (3), determined by the deformation parameter $\hbar$ (the Planck constant) and the semiclassical Ansatz $\chi=\sqrt{\rho} e^{-i S / A}$. Moreover, for the classically inaccessible regions simulated by analytical continuation of the Planck constant to pure imaginary values, $\hbar \rightarrow i \hbar$, instead of (4) we have

$$
i \psi_{\imath}+\psi_{x x}-U \psi=\left(1+\hbar^{2}\right) \frac{|\psi|_{x x}}{|\psi|} \psi
$$

Written in terms of the two real functions $Q^{ \pm}=\sqrt{\rho} e^{ \pm S / \hbar}$, this becomes the pair of decoupled diffusion-antidiffusion equations [1]

$$
\pm \hbar Q_{t}^{ \pm}-\hbar^{2} Q_{x x}^{ \pm}-U Q^{ \pm}=0
$$

\section{Quantization of the dispersionless NLS equation}

The semiclassical limit has recently been applied to the defocusing NLS equation [3]

$$
i \hbar \chi_{t}+\hbar^{2} \chi_{x x}-2 \gamma^{2}|\chi|^{2} \chi=0
$$

and provides an analytical tool to describe shock waves in nonlinear optics. Decomposing the wave function $\chi=\sqrt{\rho} e^{-i S / \hbar}$ one derives quantum deformation of the 
Hamilton-Jacobi equation (1) by the quantum potential, or after differentiation in the space coordinate and the substitution $v=-2 S_{x}$, the Madelung fluid

$$
v_{t}+v v_{x}=-2\left[2 \gamma^{2} \rho-\hbar^{2} \frac{(\sqrt{\rho})_{x x}}{\sqrt{\rho}}\right]_{x}, \quad \rho_{t}+(\rho v)_{x}=0 .
$$

In the formal semiclassical limit $\hbar \rightarrow 0$, one neglects the contribution from the quantum potential $U_{q}=(\sqrt{\rho})_{x x} / \sqrt{\rho}$, and the fluid becomes the Euler system representable in the Riemann invariant form [3].

Meanwhile, in terms of $\psi=\sqrt{\rho} e^{-i S}$, the system (6) is of NLS form deformed by the quantum potential (QPNLS-I)

$$
i \psi_{t}+\psi_{x x}-2 \gamma^{2}|\psi|^{2} \psi=\left(1-\hbar^{2}\right) \frac{|\psi|_{x x}}{|\psi|} \psi
$$

and determines the formal dispersionless NLS equation

$$
i \psi_{t}+\psi_{x x}-2 \gamma^{2}|\psi|^{2} \psi=\frac{|\psi|_{x x}}{|\psi|} \psi
$$

Like the linear case (4), where classically inaccessible regions of quantum mechanics (tunnel effect) come out from the analytical continuation $\hbar \rightarrow i \hbar$, from (7) we get QPNLS-II

$$
i \psi_{t}+\psi_{x x}-2 \gamma^{2}|\psi|^{2} \psi=\left(1+\hbar^{2}\right) \frac{|\psi|_{x x}}{|\psi|} \psi
$$

or in terms of the real functions $Q^{ \pm}=\sqrt{\rho} e^{ \pm S / \hbar}$, the reaction-diffusion analogue of the NLS

$$
\pm \hbar Q_{t}^{ \pm}-\hbar^{2} Q_{x x}^{ \pm}-2 \gamma^{2} Q^{+} Q^{-} Q^{ \pm}=0 .
$$

Both the QPNLS equations (7) and (9) can be derived from Lagrangian density

$$
\mathscr{L}=\frac{i}{2}\left(\bar{\psi} \psi_{t}-\bar{\psi}_{t} \psi\right)-\bar{\psi}_{x} \psi_{x}+\left(1+\epsilon \hbar^{2}\right)\left(|\psi|_{x}\right)^{2}-\gamma^{2}|\psi|^{4}
$$

and have Hamiltonian function

$$
\mathscr{H}=\bar{\psi}_{x} \psi_{x}-\left(1+\epsilon \hbar^{2}\right)\left(|\psi|_{x}\right)^{2}+\gamma^{2}|\psi|^{4},
$$

where $\epsilon= \pm 1$ for QPNLS-II and QPNLS-I correspondingly. When $\hbar=0$, from these forms we obtain the Lagrangian and Hamiltonian densities for dispersionless NLS (8). 


\section{Lax representation and gauge equivalence}

Integrability of the NLS equation [13] allows us to derive the Lax representation for the Madelung fluid (6) and QPNLS-I (7) and to establish their gauge equivalence. The zero curvature condition $\left(J_{1}\right)_{t}-\left(J_{0}\right)_{x}+\left[J_{0}, J_{1}\right]=0$ determined by the linear problem $g_{x}=g J_{1}, g_{t}=g J_{0}$ with

$$
\begin{gathered}
J_{1}=\frac{i}{2 \hbar}\left(\begin{array}{cc}
\lambda+v / 2 & -2 \gamma \sqrt{\rho} \\
2 \gamma \sqrt{\rho} & -\lambda-v / 2
\end{array}\right), \\
J_{0}=\frac{i}{2 \hbar}\left(\begin{array}{cc}
\lambda^{2}-v^{2} / 4+\hbar^{2}(\sqrt{\rho})_{x x} / \sqrt{\rho} & 2 \gamma\left(-i \hbar \partial_{x}-\lambda+v / 2\right) \sqrt{\rho} \\
2 \gamma\left(-i \hbar \partial_{x}+\lambda-v / 2\right) \sqrt{\rho} & -\lambda^{2}+v^{2} / 4-\hbar^{2}(\sqrt{\rho})_{x x} / \sqrt{\rho}
\end{array}\right),
\end{gathered}
$$

is equivalent to the system (6). Then a new linear problem $\tilde{g}_{x}=\tilde{g} \tilde{J}_{1}, \tilde{g}_{t}=\tilde{g} \tilde{J}_{0}$ corresponds to a new function $\tilde{g}=g e^{-h(x, t) \sigma_{3}}$ with gauge transformed potentials $\tilde{J}_{\mu}=e^{h \sigma_{3}} J_{\mu} e^{-h \sigma_{3}}-\partial_{\mu} h \sigma_{3}$. Choosing $h(x, t)=i S / 2$ we get the Lax representation for QPNLS (7):

$$
\begin{gathered}
\tilde{J}_{1}=\frac{i}{2 \hbar}\left[\lambda+(1+\hbar) \frac{i}{2}\left(\ln \frac{\bar{\psi}}{\psi}\right)_{x}\right] \sigma_{3}+\frac{i \gamma}{\hbar}\left(\begin{array}{cc}
0 & -\bar{\psi} \\
\psi & 0
\end{array}\right), \\
\tilde{J}_{0}=\frac{i}{2 \hbar}\left[\lambda^{2}+\frac{1+\hbar}{4}\left(\ln \frac{\bar{\psi}}{\psi}\right)_{x}^{2}-2 \hbar \gamma^{2}|\psi|^{2}+(1+\hbar) \hbar^{2} \frac{|\psi|_{x x}}{|\psi|}\right] \sigma_{3} \\
+\frac{i \gamma}{\hbar}\left(\left[-i \hbar \partial_{x}+\lambda-(1+\hbar) \frac{i}{2}\left(\ln \frac{\bar{\psi}}{\psi}\right)_{x}\right] \psi\right. \\
0
\end{gathered}
$$

This Lax pair coincides with the Zakharov-Shabat pair only for the real value $\hbar=-1$. In this case, the contribution from the quantum potential to the right-hand side of (7) vanishes. Alternatively, for any real $\hbar$ one can make a connection to the Zakharov-Shabat problem for NLS (5) by gauge transformation as above while choosing $h=-i S / \hbar$.

To get the Lax representation for QPNLS-II (9) one can just substitute $\hbar \rightarrow$ i $\hbar$ in (12). Then the obtained pair can be gauge connected with the Zakharov-Shabat problem for (10), with two real functions $Q^{ \pm}$.

\section{Bilinear representation}

The bilinear form for QPNLS-II, (9), is similar to the NLS form but with more complicated substitution. The solution is given in terms of three real functions $g^{ \pm}, f$,

$$
\psi=\frac{\left(g^{+}\right)^{(1-i \hbar) / 2}\left(g^{-}\right)^{(1+i \hbar) / 2}}{f}, \quad \bar{\psi}=\frac{\left(g^{+}\right)^{(1+i h) / 2}\left(g^{-}\right)^{(1-i \hbar) / 2}}{f},
$$


satisfying the bilinear equations

$$
\left( \pm \hbar D_{\ell}-\hbar^{2} D_{x}^{2}\right)\left(g^{ \pm} \cdot f\right)=0 \text { and } \hbar^{2} D_{x}^{2}(f \cdot f)=2 \gamma^{2} g^{+} g^{-} \text {. }
$$

Then for the density we have $|\psi|^{2}=\left(\hbar^{2} / \gamma^{2}\right)(\ln f)_{x x}$. The one-soliton solution is given by $g^{ \pm}=\gamma^{-1} e^{\eta_{1}^{ \pm}}, f=1+e^{\phi_{11}} e^{\eta_{1}^{+}+\eta_{1}^{-}}, e^{\phi_{11}}=\left(k_{1}^{+}+k_{1}^{-}\right)^{-2}$ or in terms of $k=\left(k_{1}^{+}+k_{1}^{-}\right) / 2, v=k^{-}-k^{+}$, the envelope soliton form

$$
\psi=\frac{k}{\gamma} \frac{\exp \left(i\left[v x / 2-\left(k^{2}+v^{2} / 4\right) t+\xi_{0}\right]\right)}{\cosh \left(k\left(x-v t-a_{0}\right) / \hbar\right)} .
$$

It is worthy of note that in contrast to the usual semiclassical expansion, when the phase is the fast oscillating function, in our case only the envelope depends on $\hbar$ and for decreasing $\hbar$ the function becomes sharper.

\section{A black hole on a hyperbolic constant curvature surface}

Let us consider two-dimensional metrics of the form

$$
d s^{2}=g_{\mu \nu} d x^{\mu} d x^{\nu}=\hbar^{2} Q_{x}^{+} Q_{x}^{-} d t^{2}+\hbar\left(Q^{+} Q_{x}^{-}-Q_{x}^{+} Q^{-}\right) d x d t-Q^{+} Q^{-} d x^{2}
$$

Then, given $Q^{ \pm}(x, t)$ satisfying (10), scalar curvature is reduced to the constant negative value $R=g^{\mu \nu} R_{\mu \nu}=-8 \gamma^{2}$. In this case functions the $Q^{ \pm}$and their space derivatives form the orthonormal moving frame in the tangent plane to the one sheet hyperboloid $S L(2, R) / O(1,1)[7,10,12]$. In terms of a solution $\psi$ of QPNLS-II (9), the components of the metric are given by

$$
g_{00}=\left(1+\epsilon \hbar^{2}\right)\left(|\psi|_{x}\right)^{2}-\bar{\psi}_{x} \psi_{x}, \quad g_{01}=\frac{i}{2}\left(\bar{\psi}_{x} \psi-\bar{\psi} \psi_{x}\right), \quad g_{11}=-|\psi|^{2} .
$$

Comparing the Hamiltonian (11) with the metric (14) we find that the dispersive part of the energy has geometrical meaning in terms of the time component $g_{00}=-\epsilon_{00}$. From the inequality $\left|\psi_{x}\right|^{2} \geq\left(|\psi|_{x}\right)^{2}$ we conclude $\epsilon_{0} \geq-\epsilon \hbar^{2}\left(|\psi|_{x}\right)^{2}$ and is nonnegative for QPNLS-I $(\epsilon=-1)$. In contrast, for QPNLS-II $(\epsilon=1)$ it can well be negative valued. Then at the space-time points where dispersion changes the sign, the component $g_{00}$ vanishes, which indicates the existence of causal singularities and black hole phenomena. For example, the one-soliton solution (13) gives $g_{00}=$ $\left[k^{2} \tanh ^{2} \frac{k}{h}\left(x-v t-a_{0}\right)-\frac{v^{2}}{4}\right]|\psi|^{2}$, and when it is propagating with bounded velocity $|v| \leq 2|k|$ it has two symmetrical points located at distance $\pm \frac{h}{k} \tanh ^{-1} \frac{v}{2 k}$ from the soliton center where $g_{00}=0$. These points correspond to the event horizon and the soliton (13) to the black hole [8] with analytical Kruskal-Szekeres causal structure [5]. The first three integrals, mass, momentum and energy,

$$
M=\frac{2 \hbar}{\gamma^{2}}|k|, \quad P=M v \quad \text { and } \quad E=\frac{M v^{2}}{2}+\frac{\gamma^{4}}{6 \hbar^{2}} M^{3},
$$



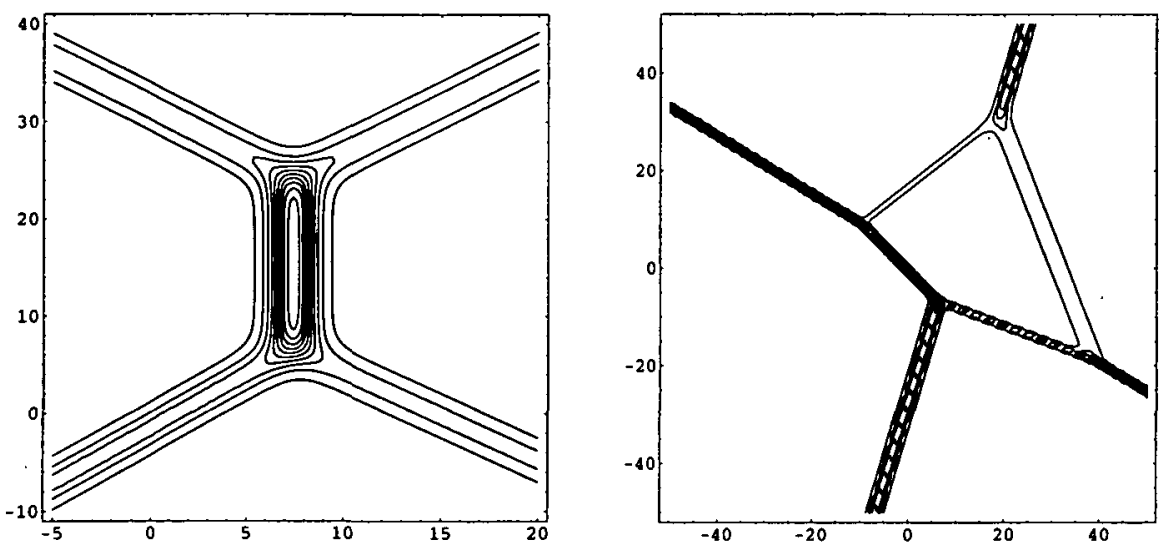

FIGURE 1. Contour plot of (a) soliton resonance; (b) four vertex interaction.

allow one to interpret the soliton as a non-relativistic particle. But existence of the positive rest energy $E_{0}=E(v=0)=4 \hbar|k|^{3} / 3 \gamma^{2}$ follows from $E=\hbar|k|\left(v^{2}+4 k^{2} / 3\right) / \gamma^{2}$, allowing decay for a soliton at rest. In fact, constructing a two-soliton solution we find a reach resonance phenomenology of soliton interactions (Figures 1 (a)-(b)) [11, 12].

\section{QPNLS with derivative nonlinearity}

6.1. Bilinear form Now we consider Derivative NLS perturbed by quantum potential. We know two integrable cases, QPDNLS,

and QPDNLS ,

$$
i \psi_{t}+\psi_{x x}+i\left(|\psi|^{2} \psi\right)_{x}=\left(1+\epsilon \hbar^{2}\right) \frac{|\psi|_{x x}}{|\psi|} \psi
$$

$$
i \psi_{t}+\psi_{x x}-i|\psi|^{2} \psi_{x}=\left(1+\epsilon \hbar^{2}\right) \frac{|\psi|_{x x}}{|\psi|} \psi
$$

For $\epsilon=-1$ one can again connect them to the standard form of DNLS equations $[4,9]$. This is why we only treat the case $\epsilon=1$ below. Then applying the same procedure, decomposing $\psi=\exp (R-i S)$ and introducing $Q^{ \pm}=\exp (R \pm S / \hbar)$ we get a "dissipative" version (derivative reaction-diffusion) of the DNLS equations

$$
\hbar Q_{t}^{ \pm}= \pm \hbar^{2} Q_{x x}^{ \pm}-\hbar\left(Q^{+} Q^{-} Q^{ \pm}\right)_{x}
$$

and

$$
\hbar Q_{t}^{ \pm}= \pm \hbar^{2} Q_{x x}^{ \pm}+\hbar Q^{+} Q^{-} Q_{x}^{ \pm}
$$


Each of these equations admits the Hirota representation allowing one to bilinearize (15) and (16). In terms of four real functions we have the bilinear system

$$
\begin{aligned}
\left( \pm \hbar D_{t}-\hbar^{2} D_{x}^{2}\right)\left(g^{ \pm} \cdot f^{ \pm}\right) & =0, \\
\hbar^{2} D_{x}^{2}\left(f^{+} \cdot f^{-}\right)-\frac{a-b}{2} \hbar D_{x}\left(g^{+} \cdot g^{-}\right) & =0, \\
\hbar D_{x}\left(f^{+} \cdot f^{-}\right)-\frac{a+b}{2}\left(g^{+} \cdot g^{-}\right) & =0 .
\end{aligned}
$$

The Painlevé test [2] restricts values of the parameters $a$ and $b$ by the condition $a b=0$. For Case $1(a=0, b=1)$, a solution of this system provides the solution of $\mathrm{QPDNLS}_{1}$-II $(15)(\epsilon=1)$, according to the relation

$$
\psi=\frac{\left(g^{+}\right)^{(1-i \hbar) / 2}\left(g^{-}\right)^{(1+i \hbar) / 2}}{\left(f^{+}\right)^{(1+3 i \hbar) / 2}\left(f^{-}\right)^{(1-3 i \hbar) / 2}}
$$

while for Case $2(a=1, b=0)$, the solution of QPDNLS - II $(16)(\epsilon=1)$ is

$$
\psi=\left(\frac{g^{+}}{f^{+}}\right)^{(1-i \hbar) / 2}\left(\frac{g^{-}}{f^{-}}\right)^{(1+i \hbar) / 2}
$$

6.2. Chiral soliton as black hole For the one-soliton solution we have

$$
g^{ \pm}=e^{\eta_{1}^{ \pm}}, \quad f^{ \pm}=1+e^{\phi_{11}^{ \pm}} e^{\eta_{1}^{+}+\eta_{1}^{-}}, \quad e^{\phi_{11}^{ \pm}}= \pm \frac{a k_{1}^{ \pm}+b k_{1}^{\mp}}{2\left(k_{1}^{+}+k_{1}^{-}\right)^{2}}
$$

where $\hbar \eta_{1}^{ \pm}=k_{1}^{ \pm} x \pm\left(k_{1}^{ \pm}\right)^{2} t+\eta_{0}^{ \pm}$. The regularity condition requires $k_{1}^{+} \leq 0, k_{1}^{-} \geq 0$ in Case 1 , and $k_{1}^{+} \geq 0, k_{1}^{-} \leq 0$ in Case 2 . Then we have density

$$
|\psi|^{2}=\frac{2 k^{2}}{\sqrt{v^{2}-k^{2}} \cosh \frac{k}{h}\left(x-v t-a_{0}\right)+|v|},
$$

where $k=k_{1}^{+}+k_{1}^{-}, v=k_{1}^{-}-k_{1}^{+}$and the velocity bounded from below $v^{2}>k^{2}$ obeys $v>0$ for Case $1\left(\right.$ QPDNLS $\left._{1}\right)$ and $v<0$ for Case $2\left(\right.$ QPDNLS $\left._{2}\right)$. So we find that our solitons are chiral, since they propagate only in one direction. The chiral property influences the position of the event horizon of the black hole corresponding to the soliton (17). In fact, calculating the metric tensor component $g_{00}$ for the one-soliton solution [6] we find that it vanishes at the horizon only if velocity is also bounded from above. Moreover, due to there being only one direction of motion, in contrast to the QPNLS from the previous section with a symmetrical pair of horizons, we have now only a single horizon which we call the chiral event horizon. 

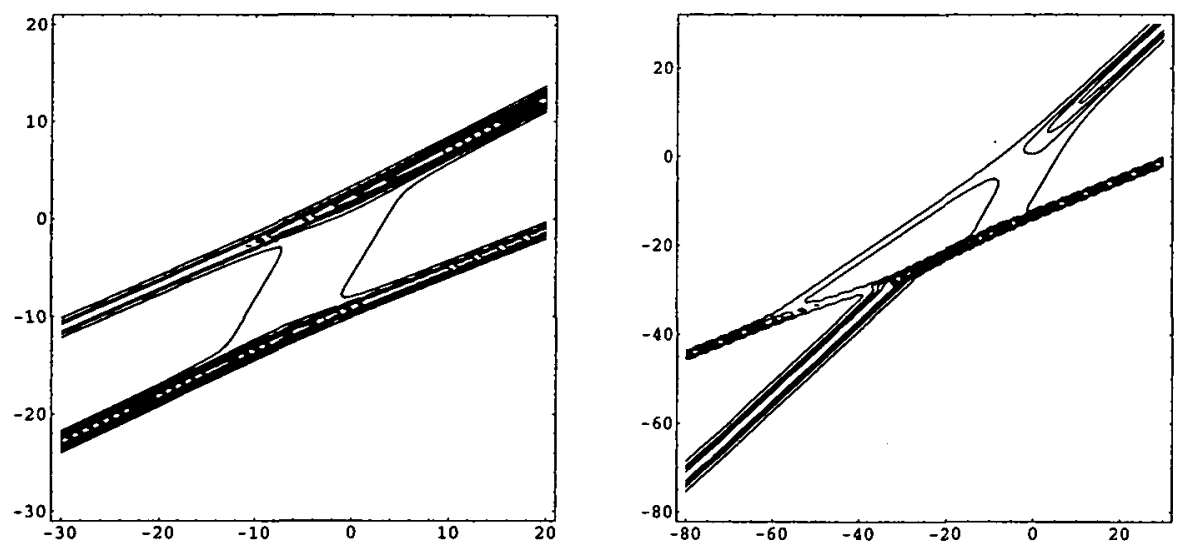

FigURE 2. Contour plot of (a) solitons exchange-type collision; (b) four vertex interaction.

6.3. Resonance state of solitons For the two-soliton solution we have

$$
\begin{aligned}
& g^{ \pm}=e^{\eta_{1}^{ \pm}}+e^{\eta_{2}^{ \pm}}+\alpha_{1}^{ \pm} e^{\eta_{2}^{+}+\eta_{2}^{-}+\eta_{1}^{ \pm}}+\alpha_{2}^{ \pm} e^{\eta_{1}^{+}+\eta_{1}^{-}+\eta_{2}^{ \pm}}, \\
& f^{ \pm}=1+\sum_{i, j=1}^{2} e^{\phi_{i j}^{ \pm}} e^{\eta_{i}^{+}+\eta_{j}^{-}}+\beta^{ \pm} e^{\eta_{1}^{+}+\eta_{1}^{-}+\eta_{2}^{+}+\eta_{2}^{-}},
\end{aligned}
$$

where $\eta_{i}^{ \pm}=k_{i}^{ \pm} x \pm\left(k_{i}^{ \pm}\right)^{2} t+\eta_{i 0}^{ \pm}, k_{i j}^{n m} \equiv\left(k_{i}^{n}+k_{j}^{m}\right)$ and

$$
\begin{gathered}
\alpha_{1}^{ \pm}=\mp \frac{(a-b)}{2} \frac{k_{2}^{\mp}\left(k_{1}^{ \pm}-k_{2}^{ \pm}\right)^{2}}{\left(k_{22}^{+-}\right)^{2}\left(k_{12}^{ \pm \mp}\right)^{2}}, \quad \alpha_{2}^{ \pm}=\mp \frac{(a-b)}{2} \frac{k_{1}^{\mp}\left(k_{1}^{ \pm}-k_{2}^{ \pm}\right)^{2}}{\left(k_{11}^{+-}\right)^{2}\left(k_{21}^{ \pm \mp}\right)^{2}}, \\
\beta^{ \pm}=\frac{\left(k_{1}^{+}-k_{2}^{+}\right)^{2}\left(k_{1}^{-}-k_{2}^{-}\right)^{2}}{4\left(k_{11}^{+-} k_{12}^{+-} k_{21}^{+-} k_{22}^{+-}\right)^{2}}\left(a^{2} k_{1}^{ \pm} k_{2}^{ \pm}+b^{2} k_{1}^{\mp} k_{2}^{\mp}\right), \\
e^{\phi_{i i}^{ \pm}}= \pm \frac{a k_{i}^{ \pm}+b k_{i}^{\mp}}{2\left(k_{i i}^{+-}\right)^{2}}, \quad e^{\phi_{i j}^{+}}=\frac{a k_{i}^{+}+b k_{j}^{-}}{2\left(k_{i j}^{+-}\right)^{2}}, \quad e^{\phi_{i j}^{-}}=-\frac{b k_{i}^{+}+a k_{j}^{-}}{2\left(k_{i j}^{+-}\right)^{2}} .
\end{gathered}
$$

The regularity conditions are now the same as for one soliton $k_{i}^{+} \leq 0, k_{i}^{-} \geq 0$ in Case 1 , and $k_{i}^{+} \geq 0, k_{i}^{-} \leq 0$ in Case 2. This solution describes a collision of two solitons propagating in the same direction and at some parameter values creates the resonance states (Figures 2 (a)-(b)).

\section{Acknowledgements}

One of the authors (O.P.) would like to thank the Institute of Mathematics, Academia Sinica, Taipei for support. 


\section{References}

[1] G. Auberson and P. C. Sabatier, "On a class of homogeneous nonlinear Schrödinger equations", J. Math. Phys. 35 (8) (1994) 4028-4040.

[2] P. A. Clarkson and C. M. Cosgrove, "Painlevé analysis of the nonlinear Scrödinger family of equations", J. Phys. A 20 (1987) 2003-24.

[3] S. Jin, C. D. Levermore and D. W. McLaughlin, "The semiclassical limit of the defocusing NLS hierarchy", Comm. Pure Appl. Math. 52 (5) (1999) 613-654.

[4] D. J. Kaup and A. C. Newell, "An exact solution for a derivative nonlinear Scrödinger equation", J. Math. Phys. 19 (1978) 789-801.

[5] M. D. Kruskal, "Maximal extension of Schwarzschild metric", Phys. Rev. (2) 119 (1960) 17431745.

[6] J.-H. Lee, C.-K. Lin and O. K. Pashaev, "Equivalence relation and bilinear representation for derivative nonlinear Scrödinger type equations", in Proceeding of Workshop on Nonlinearity, Integrability and all that: Twenty years after NEEDS'79 (eds. M. Boiti, L. Martina, F. Pempinelli, B. Prinari and G. Soliani), (World Scientific, Singapore, 2000) 175-181.

[7] L. Martina, O. K. Pashaev and G. Soliani, "Integrable dissipative structures in the gauge theory of gravity", Classical Quantum Gravity 14 (1997) 3179-3186.

[8] L. Martina, O. K. Pashaev and G. Soliani, "Bright solitons as black holes", Phys. Rev. $D$ (3) 58 (1998) 084025.

[9] A. Nakamura and H. H. Chen, "Multisoliton solutions of à derivative nonlinear Scrödinger equation", J. Phys. Soc. Japan 49 (1980) 813-816.

[10] O. K. Pashaev, "Integrable models as constrained topological gauge theory. Constrained dynamics and quantum theory", Nucl. Phys. B Proc. Suppl. 57 (1997) 338-341.

[11] O. K. Pashaev and J.-H. Lee, "Soliton resonances, black holes and Madelung fluid", J. Nonl. Math. Phys. Supplement Vol. 8 (2001) 230-234.

[12] O. K. Pashaev and J.-H. Lee, "Resonance NLS solitons as black holes in Madelung fluid", hepth/9810139.

[13] V. E. Zakharov and A. B. Shabat, "Exact theory of two-dimensional self-focusing and onedimensional self-modulation of waves in nonlinear media", Soviet Physics JETP 34 (1) (1972) $62-69$. 